\title{
Aerosol agitation: Quantifying the hydrodynamic stressors on particulates encapsulated in small droplets
}

\author{
Oliver McRae $\odot,{ }^{1}$ Kenneth R. Mead $\odot,{ }^{2}$ and James C. Bird $\oplus^{1, *}$ \\ ${ }^{1}$ Department of Mechanical Engineering, Boston University, Boston, Massachusetts 02215, USA \\ ${ }^{2}$ Division of Field Studies and Engineering, National Institute for Occupational Safety and Health, Centers \\ for Disease Control and Prevention, Cincinnati, Ohio 45226, USA
}

(Received 30 August 2020; accepted 11 February 2021; published 8 March 2021)

\begin{abstract}
Lower respiratory tract infections originate from multiple aerosol sources, varying from droplets erupting from bursting bubbles in a toilet or those produced by human speech. A key component of the aerosol-based infection pathway-from source to potential host-is the survival of the pathogen during aerosolization. Due to their finite-time instability, pinch-off processes occurring during aerosolization have the potential to rapidly accelerate the fluid into focused regions of these droplets, stress objects therein, and if powerful enough, disrupt biological life. However, the extent that a pathogen will be exposed to damaging hydrodynamic stressors during the aerosolization process is unknown. Here we compute the probability that particulates will be exposed to a hydrodynamic stressor during the generation of droplets that range in size from one to 100 microns. For example, particulates in water droplets less than $5 \mu \mathrm{m}$ have a $50 \%$ chance of being subjected to an energy dissipation rate in excess of $10^{11} \mathrm{~W} / \mathrm{m}^{3}$, hydrodynamic stresses in excess of $10^{4} \mathrm{~Pa}$, and strain rates in excess of $10^{7} \mathrm{~s}^{-1}$, values known to damage certain biological cells. Using a combination of numerical simulations and self-similar dynamics, we show how the exposure within a droplet can be generally predicted from its size, surface tension, and density, even across different aerosolization mechanisms. Collectively, these results introduce aerosol agitation as a potential factor in pathogen transmission and implicate the pinch-off singularity flow as setting the distribution of hydrodynamic stressors experienced within the droplet.
\end{abstract}

DOI: 10.1103/PhysRevFluids.6.L031601

Like many communicable diseases, lower respiratory tract infections are spread when small pathogen-laden drops, which span both droplets and aerosols, are transported from a source to a host. Aerosol-transmitted pathogens are highly diverse, encompassing viruses, fungi, and bacteria with varied size, structure, and transmission rates. Distributions of varying-sized droplets potentially containing these pathogens can be generated from both respiratory [1-4] and environmental sources [5-7] [Fig. 1(a)]. The size and number of aerosols play a key role in pathogen transmission, as they dictate the aerosol dispersal, persistence time in air, ability to be inhaled by particular regions of the lungs, and pathogen load [8]. However, the number of pathogens within droplets is secondary if the majority of the pathogens are damaged to the point where they are noninfective. Previous studies have shown that a fraction of microorganisms dispersed by droplets can be rendered nonviable from factors such as temperature and humidity $[9,10]$. Here we postulate that under certain conditions pathogen damage may also occur from hydrodynamic agitation.

*jbird@bu.edu 

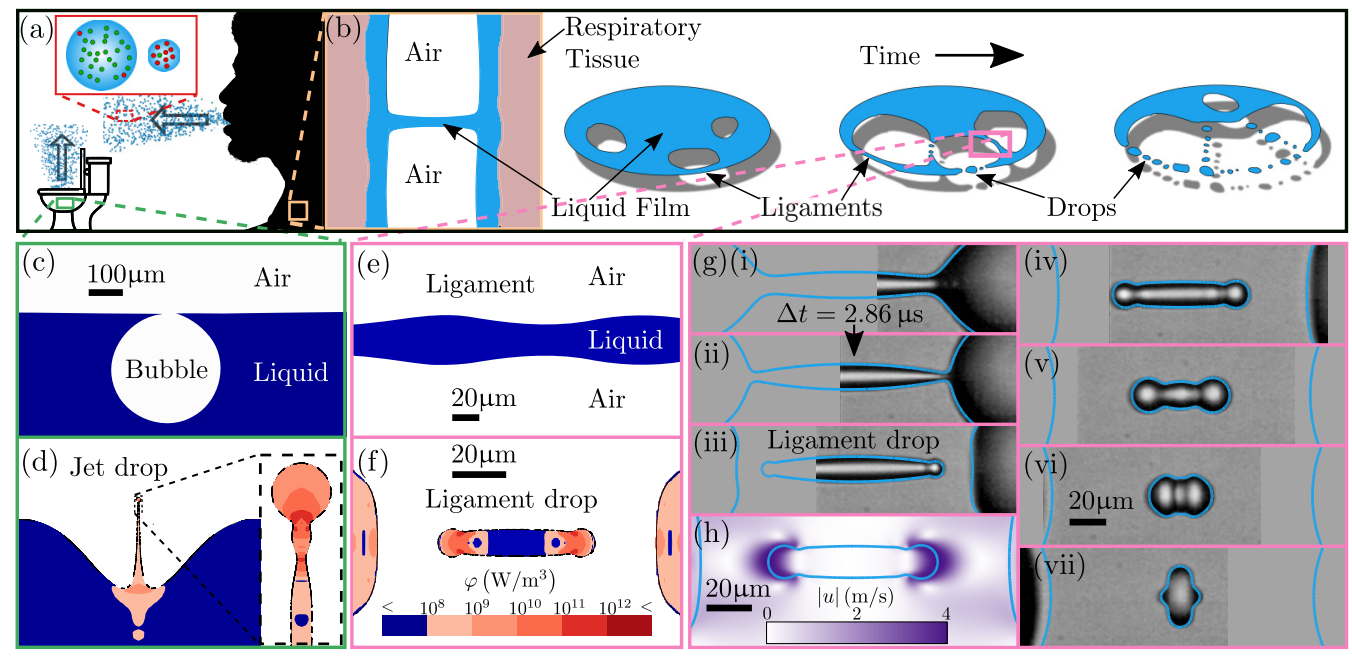

FIG. 1. Capillary forces generate energy dissipation rates $(\varphi)$ during drop formation high enough to damage pathogens. (a) A schematic illustrates that pathogens from respiratory and environmental sources can be transferred in drops. Yet whether or not the pathogens are infectious (inset: green-infectious, red-noninfectious) may depend more on drop size than source aerosolization mechanism. (b) One mechanism to generate respiratory aerosols occurs when liquid bridges develop within airways. As the air stretches these thin films, holes nucleate and combine to form ligaments, which then break up into drops. (c) Aerosols also form when bubbles rise to a surface and rupture. (d) Numerical simulations of the rupture can calculate the EDR from hydrodynamic agitation during jet drop generation. [(e) and (f)] Simulations of a cylindrical ligament breakup reveal a comparable EDR when a similarly sized drop is generated. (g)[(i)-(vii)] High-speed images from experiments $(\Delta t=2.86 \mu \mathrm{s})$ during ligament drop $\left(D_{d}=28 \mu \mathrm{m}\right)$ breakup closely match interface profiles from corresponding numerical simulations (blue lines). (h) The velocity field from numerical simulationscorresponding to experimental frame (iv) - highlights large spatial gradients as the ligament drop retracts.

The idea that intense fluid agitation can damage microorganisms is well documented [11]. Chronic, minutes-long agitation of the measles and mumps viruses has shown a virus-dependent reduction in infectivity - through degradation of the lipid bilayer of the viral envelope-at strain rates less than $10^{4} \mathrm{~s}^{-1}$ [12-14]. Acute agitation of the bacteria Lactococcus lactis experimentally showed a significant reduction in survival at fluid strain rates greater than $10^{5} \mathrm{~s}^{-1}$ [15]. The yeast Saccharomyces cerevisiae was rendered nonviable at strain rates greater than $10^{6} \mathrm{~s}^{-1}$ as well as at shear stresses greater than $2800 \mathrm{~Pa}[11,16]$. Meanwhile the bacteria Escherichia coli showed no reduction in viability at shear stresses less than $500 \mathrm{~Pa}$, a $10 \%$ reduction with no cell lysis at $1300 \mathrm{~Pa}$, and an $80 \%$ reduction with lysis at $1800 \mathrm{~Pa}$ [11]. In the context of animal and insect cells used in bioreactors [17]; cell-damaging fluid agitation is typically quantified as the scalar-valued energy dissipation rate (EDR) $\varphi$ :

$$
\varphi=\mu\left[\nabla u+(\nabla u)^{T}\right]: \nabla u,
$$

where $\mu$ is the liquid viscosity and $\nabla u$ the spatial gradient of the velocity field [18-20]. Use of the scalar $\operatorname{EDR} \varphi$ is advantageous as it embodies both the shear and extensional components of a three-dimensional fluid flow, components separately implicated in damage to biological cells [21]. Furthermore, a scalar-valued characteristic shear rate and shear stress can be defined from the EDR as $\dot{\gamma}=\sqrt{\varphi / \mu}$ and $\tau=\sqrt{\mu \varphi}$, respectively.

Within bioreactors, lethal EDRs are attributed to bursting bubbles and ruptured films [22,23]. Here the focus has been on the hydrodynamic agitation in the bulk, not within the droplets that often develop during film and bubble rupture $[24,25]$. Yet, it is precisely these droplets that are a source 


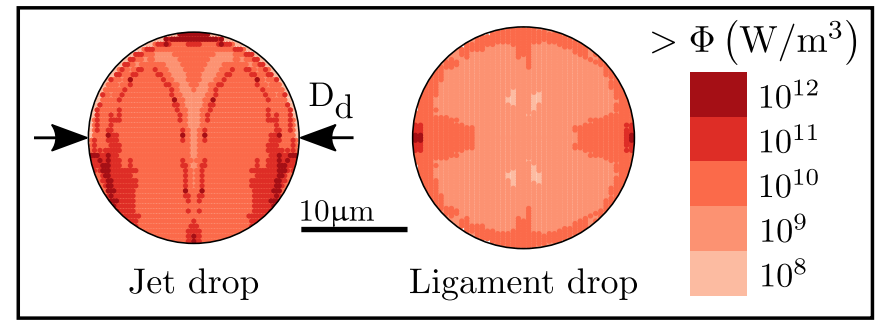

FIG. 2. Lagrangian particle tracking to uncover the maximum energy dissipation rate $\Phi$ at a particular location. By tracking fluid parcels in the simulations, an exposure map can be computed of the maximum EDR $\Phi$ experienced at each material point within the drop. Here the jet and ligament drops have diameters approximately $D_{d} \approx 20 \mu \mathrm{m}$.

of both respiratory and environmental pathogen transport. For example, studies have suggested that breath aerosols develop when films of respiratory tract lining fluid form across expanding bronchioles [26]. As the film stretches, it thins and forms holes that retract and create ligaments of fluid that pinch-off into drops [Fig. 1(b)] [27]. Similarly, flushing toilets have been shown to generate pathogen-bearing aerosols from bubble rupture [28]. The sloshing of the contaminated toilet water entrains air bubbles that rise to the surface [Fig. 1(c)]; these bubbles, on rupture, produce an upward jet that can pinch-off into jet drops [Fig. 1(d)]. For both of these examples - and in fact most aerosolization processes-pinch-off is an existential process of droplet production [29]. It is also an acute source of intense EDRs, the parameter previously associated with cell damage. Therefore, we propose that the hydrodynamics that form certain aerosols may generate an EDR so large that it would be expected to damage biological cells encapsulated in those small droplets. This idea may initially appear counterintuitive because it suggests that the same mechanism by which pathogens are successfully dispersed also destroys them. However, we are not suggesting that all droplets from these mechanisms would damage biological cells. Furthermore, we propose that not all encapsulated cells would experience uniformly damaging stressors, due to spatial variability in the EDR experienced.

To test our hypothesis, we conduct multiphase fluid dynamic simulations with a focus on two drop-generating scenarios: bubble rupture and ligament breakup. Solutions to the axisymmetric, incompressible Navier-Stokes equations are computed in the absence of gravity, using the volume of fluid method with the open-source solver Gerris [30]. Gerris is particularly suitable for interfacial fluid phenomena as it uses an automatically generated and adaptively refined quadtree mesh in an axisymmetric framework to capture the finer details of the fluid flow and pinch-off dynamics. This axisymmetric framework allows for the simulation of three-dimensional space in two dimensions, radial $r$ and axial $z$ coordinates, through revolution about the axis of symmetry $(r=0)$. For easier envisaging of our simulation results, we have mirrored the results about the axis of symmetry in Figs. 1, 2, and 4 . In the case of the submerged bubble, a spherical gas cavity surrounded by a liquid rests at the surface of a quiescent liquid bath [Fig. 1(c) with vertical axis of symmetry]. The bubble with an initial radius $R$ is initiated with a hole, with a radius of $0.01 R$, at the apex of the bubble. The simulation domain is a box of base $10 R$ and height $20 R$ with free-slip, impenetrable boundary conditions. In the case of the ligament breakup, a cylindrical liquid thread with an initial radius $R$, and sinusoidal perturbation with an amplitude of $0.02 R$, is initiated in a quiescent gas to render the interface unstable [Fig. 1(e) with horizontal axis of symmetry]. Here the simulation domain is a box with sides of length $20 R$, with free-slip, impenetrable boundary conditions at the top $(r=20 R)$ and axis of symmetry $(r=0)$, and periodic boundary conditions at both ends $(z=0$ and $z=20 R)$.

We focus on ligament drops and jet drops, as they characterize a broader classification scheme for aerosol generation: those in which the flow diverges symmetrically around the pinch-off singularity and those in which the droplet is formed by flow through the singularity, respectively. Even though 
both systems are initially quiescent, surface tension rapidly deforms the interface and drives a flow that ultimately develops droplets. Comparisons between simulations and experiments on the dynamics of top jet drop formation based on the above geometry were carried out in previous work [31]. To confirm that the model assumptions were appropriate for ligament drops, we experimentally generated droplets from a thin cylindrical thread of water. This thread was formed at the opening of a pulled borosilicate glass capillary attached via a 22 gauge needle to a syringe pump. At a distance from the exit of the capillary, much greater than the thread diameter, the Rayleigh-Plateau instability caused the thread to pinch-off into large and small droplets. The smaller of the two droplets was recorded with a Photron SA5 high-speed camera at 350,000 frames per second. High-speed images highlight the rapid rearrangement of the liquid before [Figs. 1(g)(i) and 1(g)(ii)] and after pinch-off [Figs. 1(g)(iii)-1(g)(vii)], and locations of the interface are nearly indistinguishable from those in the simulations. In this paper, we consider the pinch-off process of a drop with diameter $D_{d}$ to span the dynamics surrounding the topological change over a characteristic pinch-off timescale that corresponds to the inertial-capillary timescale $\sqrt{\rho D_{d}^{3} / \gamma}$. Here $\rho$ and $\gamma$ are the liquid density and surface tension respectively, leading to a pinch-off timescale in Fig. 1 of approximately $10 \mu \mathrm{s}$. Indeed, images over this timescale display large fluid velocities and velocity gradients, especially at the ends of the retracting droplet [Fig. 1(h)].

By calculating spatial gradients in the simulated velocity field at each point in time $t$ and space $\mathbf{x}$, we can extract the instantaneous $\operatorname{EDR} \varphi(\mathbf{x}, t)$. Through Lagrangian particle tracking, we can then determine the maximum EDR that a material fluid parcel $\mathbf{X}$ is exposed to $\Phi(\mathbf{X})$ over the entire time history. Lagrangian particle tracking was performed in reverse by a custom MATLAB script using fluid flow field data imported from the numerical simulations. A uniformly spaced $50 \times 50$ particle grid was initialized over the drop to be interrogated. Note that due to the axisymmetric geometry and initial spacing, the tracking script takes into account that particles initialized at a greater radial distance $r$ have a correspondingly larger fluid parcel volume. Particles corresponding to the gas phase were then removed, and the remaining liquid phase particles advected backwards in time using the velocity components from the simulation. Note that these simulations neglect any influence between particles and the fluid flow, and thus these tracers can be proxies for encapsulated particulates provided that the particulates are sufficiently small and dilute.

Figures 1(d) and 1(f) illustrate the interface shape and instantaneous EDR that develop for a representative jet drop and ligament drop. For the flow resulting from a $320-\mu \mathrm{m}$ diameter bubble and a 30- $\mu$ m diameter ligament (with air and water properties), the EDR ranges from less than $10^{6}$ $\mathrm{W} / \mathrm{m}^{3}$ (blue in Fig. 1) to over $10^{12} \mathrm{~W} / \mathrm{m}^{3}$ (maroon in Fig. 1), with the highest values occurring near the drop pinch-off region. Even though the geometries of these two cases are quite distinct, the top jet drop and smallest ligament drop both have a similar diameter $D_{d} \approx 20 \mu \mathrm{m}$. With Lagrangian particle tracking, we can map the largest EDR to which various material points are subjected (Fig. 2). In both cases, the majority of the drop has been exposed to an EDR $\Phi$ that resides between $10^{9}$ $\mathrm{W} / \mathrm{m}^{3}$ and $10^{11} \mathrm{~W} / \mathrm{m}^{3}$.

To assess the role of droplet size, we perform a sweep of initial bubble and ligament diameters in the numerical setup and obtain a range of jet and ligament drop sizes. We measure these drop diameters $D_{d}$ and, using particle tracking, construct spatial EDR exposure maps similar to Fig. 2. To quantify the damage potential into a single scalar value, we compute the median volumetric exposure EDR $\Phi_{50}$, which corresponds to the EDR value exceeded by $50 \%$ of the drop volume. For the jet drop and ligament drop illustrated in Fig. 2, these values of $\Phi_{50}$ are $4.6 \times 10^{10} \mathrm{~W} / \mathrm{m}^{3}$ and $5.6 \times 10^{9} \mathrm{~W} / \mathrm{m}^{3}$, respectively (larger symbols in Fig. 3). Note that if particulates were uniformly distributed within drops, then $\Phi_{50}$ is equivalent to the minimum EDR experienced by half of the aerosolized particulates.

Our numerical simulations indicate that $\Phi_{50}$ of jet and ligament drops is highly dependent on $D_{d}$ and increases as the drop size gets smaller (Fig. 3). Indeed, the median volumetric EDR for both jet and ligament drops follows the scaling $\Phi_{50} \sim D_{d}^{-5 / 2}$. Even though the source mechanism has an effect on the prefactor, $\Phi_{50}$ typically differs by less than an order of magnitude between the jet 


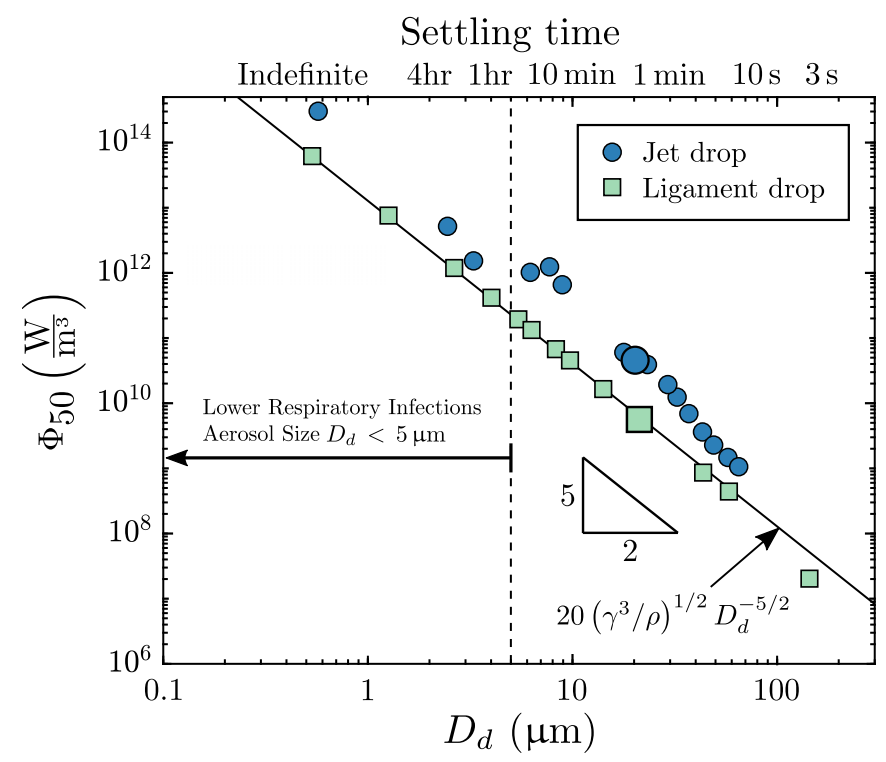

FIG. 3. While generating a drop with diameter $D_{d}$, there exists an energy dissipation rate $\Phi_{50}$ that is exceeded by $50 \%$ of the drop volume. Simulation results for both the jet (circles) and ligament (squares) breakup mechanisms suggest that the majority of fluid in the smallest drops will exceed an EDR previously known to damage bacteria and mammalian cells. Settling time is the measure of time for a drop to fall from a height of $2 \mathrm{~m}$ based on a balance of gravitational and air drag forces.

and ligament cases for the same drop size. Given the dependence of $\Phi_{50}$ on droplet size $D_{d}$, our results suggest that biological cells in the smallest droplets are most likely to be hydrodynamically damaged. These results are qualitatively consistent with experiments showing that bacterial viability decreases when aerosolized into smaller-sized droplets [32,33]. It is noteworthy that the viability independently reduced when the bacteria were aerosolized in a different media [34], and bacteria may also alter their local environment via biosecretions [35]. These observations hint that in addition to the droplet size, the liquid properties, such as surface tension and viscosity, could play a critical role in setting the hydrodynamic agitation.

To determine the effect of varying fluid properties, we look to a balance between the driving force from surface tension $\gamma$ and the resisting forces of viscosity and inertia. Given the importance of dissipation in EDR, it is natural to expect viscous forces to dominate any inertial effects in setting the median exposure EDR so that, by dimensional analysis, $\Phi_{50} \sim\left(\gamma^{2} / \mu\right) D_{d}^{-2}$. However for many drop phenomena, inertia has a dominant role when $\rho \gamma D_{d} / \mu^{2}>1$. Given that this inequality is met for the droplet conditions considered here, we might expect energy and momentum balances to be dominated by inertial and capillary effects. This balance does not necessarily imply that viscous dissipation would be negligible. For example, in the retraction of a thin film, the rate of energy dissipation is often assumed to be equivalent to the rate that surface energy is converted to kinetic energy. With this assumption, exactly half of the liberated surface energy is dissipated in the retracting rim and the energy dissipation rate is independent of viscosity [36]. Indeed, this independence of viscosity on the EDR was invoked by Cherry and Hulle when modeling cell death in thin retracting films that do not break up into droplets [22]. We consider how the EDR could scale independently from viscosity based on the single lengthscale $D_{d}$ and find

$$
\Phi_{50} \sim\left(\gamma^{3} / \rho\right)^{1 / 2} D_{d}^{-5 / 2} .
$$

This scaling (solid line in Fig. 3) agrees well with the numerical results and implies that despite viscosity being important in setting the droplet size, the value of the EDR depends predominantly 


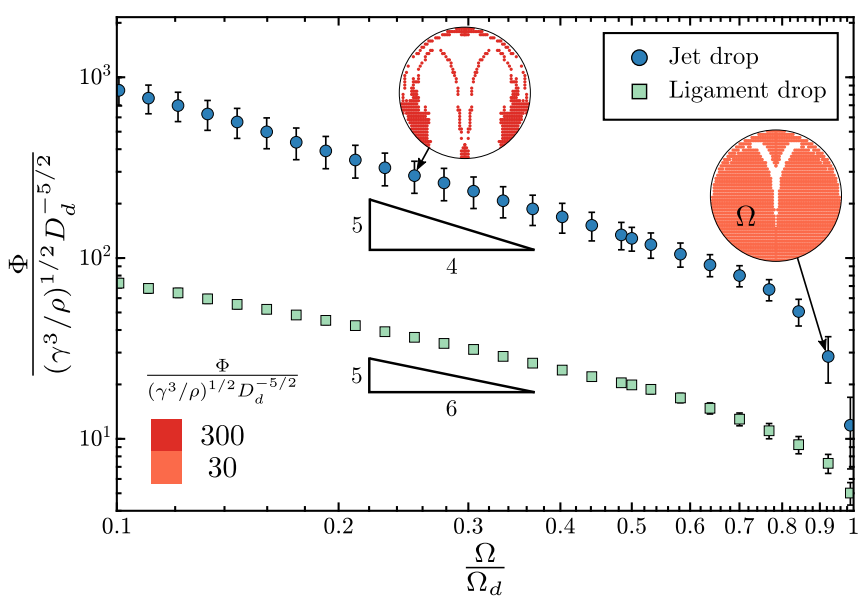

FIG. 4. Aggregate simulation data of nondimensional EDR for varying volume fractions $\Omega / \Omega_{d}$, where $\Omega_{d}$ is the volume of the droplet. Here the error bars denote the standard error from the simulations in Fig. 3.

on inertia and surface tension. Adding the prefactor to the scaling-a value of 20 and 130 for the ligament drops and jet drops respectively_provides predictive power for the exposure EDR under a range of fluid properties and drop sizes.

Calculating the probability associated with the fate of encapsulated particulates requires an understanding of how the range of exposure EDR is distributed within a single droplet. For example, most of the jet drop in Fig. 2 is exposed to an EDR that exceeds $10^{10} \mathrm{~W} / \mathrm{m}^{3}$; however, $27 \%$ of the drop volume $\Omega_{d}$ also exceeds $\Phi=10^{11} \mathrm{~W} / \mathrm{m}^{3}$. Given that Fig. 1(f) highlights that slightly after pinch-off the instantaneous EDR within the drop depends on the distance to the pinch-off singularity, we conjecture that distributions of EDR exposure also depend on proximity to this singularity. Noting that liquid pinch-off dynamics lose memory of the initial conditions and exhibit universality $[37,38]$, we construct a length-scale from the relevant parameters as $\ell \sim \gamma^{3 / 5} \rho^{-1 / 5} \Phi^{-2 / 5}$. In the absence of advection, the fraction of volume $\Omega \sim \ell^{3}$ exposed to an EDR of $\Phi$ within the droplet volume $\Omega_{d} \sim D_{d}^{3}$ is $\Omega / \Omega_{d} \sim\left[\gamma^{3} /\left(\rho \Phi^{2} D^{5}\right)\right]^{3 / 5}$ or equivalently

$$
\frac{\Phi}{\left(\gamma^{3} / \rho\right)^{1 / 2} D_{d}^{-5 / 2}} \sim\left(\frac{\Omega}{\Omega_{d}}\right)^{-\alpha}
$$

with $\alpha=5 / 6$. Note that the left-hand side of this scaling is a nondimensional EDR with a value that is equal to the scaling prefactor of Eq. (2) when $\Omega / \Omega_{d}=0.5$. For a jet drop, the instantaneous EDR would be expected to be similar near the singularity, yet the exposure EDR would be higher due to the Lagranian dynamics. If we estimate that the volume flowing through the cross-sectional area near pinch-off scales as $\Omega \sim \ell^{2} D_{d}$, then it follows that the power-law exponent in Eq. (3) would increase to $\alpha=5 / 4$.

To test these predictions, we return to the Lagrangian exposure maps for each of the simulations denoted in Fig. 3 and compute the value of $\Phi$ that is exceeded at various volume fractions $\Omega / \Omega_{d}$. We then aggregate this data to determine the mean nondimensional EDR at a given volume fraction along with its standard error (Fig. 4). At lower volume fractions, the distribution of exposed EDR indeed follow power-law relationships with exponents that are consistent with the self-similar models. At higher volume fractions, these curves deviate from the self-similar predictions, suggesting that the drop geometry is no longer negligible at these scales.

Taken together, our study highlights the potential for hydrodynamic agitation to damage certain biological cells during aerosol formation and provides evidence that not all cells that are encapsulated will be stressed to the same extent. Previous work has demonstrated that hydrodynamic 
stressors, such as strain rate, shear stress, and EDR can damage microbes, with specific values depending on the particular microbe and conditions. Moreover, it has been speculated that the aerosolization process itself could be responsible for loss in viability observed in certain aerosolization studies $[34,39,40]$. Our results quantify this process and identify the fraction of material within a drop that has been exposed to a particular EDR during jet drop and ligament drop formation, a value that can be converted to a characteristic shear stress or strain rate.

Even though our results are indifferent to the precise mechanisms by which microbe damage might occur, they confirm that this damage is not due to a temperature increase associated with hydrodynamic agitation. We note that the local rate of temperature change can be approximated with the thermal energy equation as the energy dissipation rate divided by the product of the liquid density and specific heat $c_{p}$. A quick scaling reveals that the change in temperature scales as $\Delta T \sim$ $\gamma /\left(\rho c_{p} D_{d}\right)$, where we have used the EDR scaling in Eq. (2) integrated over the characteristic pinchoff timescale $\sqrt{\rho D_{d}^{3} / \gamma}$. For all water drop sizes that we consider, the temperature change would be less than $1^{\circ} \mathrm{C}$, which would be insignificant to any microbe, and therefore we conclude that any susceptibility to EDR would occur from mechanical means.

Recognizing that an acute hydrodynamic damage threshold for viruses has not been reported in the literature, we provide a simple elastic shell model to give a rough estimate for a damage threshold of viruses based on the irreversible deformation of the viral capsid [41]. For a larger virus such as influenza, this model suggests an acute threshold EDR for damage on the order of $10^{11}$ $\mathrm{W} / \mathrm{m}^{3}$, which based on our results, suggests that the infectivity of some respiratory viruses may be affected by the fluid agitation created during the formation of micron-scale aerosols. It should be emphasized that we are not suggesting that all susceptible viruses necessarily would be neutralized by this process, but rather that the viable fraction of the aerosolized population would decrease. As this viable fraction decreases, so, too, does the infectious load, requiring on average a longer exposure time to lead to an infection.

Although there are several ways in which respiratory and environmental aerosols can be produced, the derived scaling relations suggest that the dynamics well before pinch-off are important in setting the droplet size and flow about this singularity but otherwise are largely irrelevant in determining the exposure EDR $\Phi$. A similar singularity occurs during droplet coalescence and therefore these ideas may extend to droplet deposition and impinger-based pathogen sampling $[37,44]$. In addition, past research demonstrated that a saline spray can reduce the aerosol concentration emitted by a superspreader, a result that was linked to the increase in the surface tension of the lining fluid [45]. Our scaling results suggest that increasing surface tension may be doubly productive as it would also increase the hydrodynamic agitation within droplets. Finally, it is conceivable that variations in a tolerance to intense fluid agitation across microbes may contribute to certain pathogens being better able to survive aerosolization and thus be more contagious than others. Driven by this notion, we have calculated in a probabilistic manner the expected stressors an encapsulated particulate would be exposed to during the droplet formation process, a key step to bring us closer towards the a priori determination of hydrodynamic-affected aerosol microbial viability, and thus improve the modeling of aerosol-based disease transmission.

\section{ACKNOWLEDGMENTS}

The findings and conclusions in this report are those of the authors and do not necessarily represent the official position of the National Institute for Occupational Safety and Health, Centers for Disease Control and Prevention. This work was supported by the National Science Foundation under Grant No. 1351466. The authors thank the NSF INTERN program for providing funds for O.M. to develop these ideas while on an internship at the CDC in 2017-2018. We also thank P. Barbone, J. Mizgerd, J. Bennett, and G. Lee for helpful discussions. 
[1] R. S. Papineni and F. S. Rosenthal, The size distribution of droplets in the exhaled breath of healthy human subjects, J. Aerosol Med. 10, 105 (1997).

[2] K. P. Fennelly, J. W. Martyny, K. E. Fulton, I. M. Orme, D. M. Cave, and L. B. Heifets, Cough-generated aerosols of mycobacterium tuberculosis: A new method to study infectiousness, Am. J. Respir. Crit. Care Med. 169, 604 (2004).

[3] S. Mubareka, A. C. Lowen, J. Steel, A. L. Coates, A. García-Sastre, and P. Palese, Transmission of influenza virus via aerosols and fomites in the guinea pig model, J. Infect. Dis. 199, 858 (2009).

[4] R. M. Jones and L. M. Brosseau, Aerosol transmission of infectious disease, J. Occup. Environ. Med. 57, 501 (2015).

[5] T. J. Dondero Jr, R. C. Rendtorff, G. F. Mallison, R. M. Weeks, J. S. Levy, E. W. Wong, and W. Schaffner, An outbreak of legionnaires' disease associated with a contaminated air-conditioning cooling tower, N. Engl. J. Med. 302, 365 (1980).

[6] D. B. Jernigan, J. Hofmann, M. S. Cetron, J. Nuorti, B. Fields, R. Benson, R. Breiman, H. Lipman, R. Carter, C. Genese et al., Outbreak of legionnaires' disease among cruise ship passengers exposed to a contaminated whirlpool spa, Lancet 347, 494 (1996).

[7] Y. S. Joung, Z. Ge, and C. R. Buie, Bioaerosol generation by raindrops on soil, Nat. Commun. 8, 14668 (2017).

[8] J. Gralton, E. Tovey, M.-L. McLaws, and W. D. Rawlinson, The role of particle size in aerosolised pathogen transmission: A review, J. Infect. 62, 1 (2011).

[9] S. Webb, Factors affecting the viability of air-borne bacteria. I. Bacteria aerosolized from distilled water, Can. J. Microbiol. 5, 649 (1959).

[10] P. Hambleton, M. Broster, P. Dennis, R. Henstridge, R. Fitzgeorge, and J. Conlan, Survival of virulent legionella pneumophila in aerosols, Epidemiol. Infect. 90, 451 (1983).

[11] H. Lange, P. Taillandier, and J.-P. Riba, Effect of high shear stress on microbial viability, J. Chem. Tech. Biotechnol. 76, 501 (2001).

[12] D. Sviben, D. Forčić, T. Kurtović, B. Halassy, and M. Brgles, Stability, biophysical properties and effect of ultracentrifugation and diafiltration on measles virus and mumps virus, Arch. Virol. 161, 1455 (2016).

[13] D. Loewe, J. Häussler, T. A. Grein, H. Dieken, T. Weidner, D. Salzig, and P. Czermak, Forced degradation studies to identify critical process parameters for the purification of infectious measles virus, Viruses 11, 725 (2019).

[14] G. Bai, J. S. Bee, J. G. Biddlecombe, Q. Chen, and W. T. Leach, Computational fluid dynamics (cfd) insights into agitation stress methods in biopharmaceutical development, Int. J. Pharmaceut. 423, 264 (2012).

[15] A. Ghandi, I. B. Powell, T. Howes, X. D. Chen, and B. Adhikari, Effect of shear rate and oxygen stresses on the survival of lactococcus lactis during the atomization and drying stages of spray drying: A laboratory and pilot scale study, J. Food Eng. 113, 194 (2012).

[16] J. Thacker, An approach to the mechanism of killing of cells in suspension by ultrasound, Biochim. Biophys. Acta 304, 240 (1973).

[17] J. J. Chalmers and F. Bavarian, Microscopic visualization of insect cell-bubble interactions. ii: The bubble film and bubble rupture, Biotechnol. Progr. 7, 151 (1991).

[18] N. Ma, K. W. Koelling, and J. J. Chalmers, Fabrication and use of a transient contractional flow device to quantify the sensitivity of mammalian and insect cells to hydrodynamic forces, Biotechnol. Bioeng. $\mathbf{8 0}$, 428 (2002).

[19] R. Godoy-Silva, M. Mollet, and J. J. Chalmers, Evaluation of the effect of chronic hydrodynamical stresses on cultures of suspensed cho-6e6 cells, Biotechnol. Bioeng. 102, 1119 (2009).

[20] W. Hu, C. Berdugo, and J. J. Chalmers, The potential of hydrodynamic damage to animal cells of industrial relevance: Current understanding, Cytotechnology 63, 445 (2011).

[21] Y. B. Bae, H. K. Jang, T. H. Shin, G. Phukan, T. T. Tran, G. Lee, W. R. Hwang, and J. M. Kim, Microfluidic assessment of mechanical cell damage by extensional stress, Lab Chip 16, 96 (2016).

[22] R. S. Cherry and C. T. Hulle, Cell death in the thin films of bursting bubbles, Biotechnol. Progr. 8, 11 (1992).

[23] J. Boulton-Stone and J. Blake, Gas bubbles bursting at a free surface, J. Fluid Mech. 254, 437 (1993). 
[24] M. A. Garcia-Briones, R. S. Brodkey, and J. J. Chalmers, Computer simulations of the rupture of a gas bubble at a gasliquid interface and its implications in animal cell damage, Chem. Eng. Sci. 49, 2301 (1994).

[25] P. L. Walls, O. McRae, V. Natarajan, C. Johnson, C. Antoniou, and J. C. Bird, Quantifying the potential for bursting bubbles to damage suspended cells, Sci. Rep. 7, 15102 (2017).

[26] G. R. Johnson and L. Morawska, The mechanism of breath aerosol formation, J. Aerosol Med. Pulm. Drug Deliv. 22, 229 (2009).

[27] E. Villermaux, Fragmentation, Annu. Rev. Fluid Mech. 39, 419 (2007).

[28] D. L. Johnson, K. R. Mead, R. A. Lynch, and D. V. Hirst, Lifting the lid on toilet plume aerosol: A literature review with suggestions for future research, Am. J. Infect. Contr. 41, 254 (2013).

[29] J. Eggers and E. Villermaux, Physics of liquid jets, Rep. Prog. Phys. 71, 036601 (2008).

[30] S. Popinet, An accurate adaptive solver for surface-tension-driven interfacial flows, J. Comput. Phys. 228, 5838 (2009).

[31] C. F. Brasz, C. T. Bartlett, P. L. L. Walls, E. G. Flynn, Y. E. Yu, and J. C. Bird, Minimum size for the top jet drop from a bursting bubble, Phys. Rev. Fluids 3, 074001 (2018).

[32] B. Lighthart* and B. T. Shaffer, Increased airborne bacterial survival as a function of particle content and size, Aerosol Sci. Technol. 27, 439 (1997).

[33] M. O. Fernandez, R. J. Thomas, N. J. Garton, A. Hudson, A. Haddrell, and J. P. Reid, Assessing the airborne survival of bacteria in populations of aerosol droplets with a novel technology, J. R. Soc. Interface 16, 20180779 (2019).

[34] B. Marthi, V. Fieland, M. Walter, and R. Seidler, Survival of bacteria during aerosolization, Appl. Environ. Microbiol. 56, 3463 (1990).

[35] S. Poulain and L. Bourouiba, Biosurfactants Change the Thinning of Contaminated Bubbles at BacteriaLaden Water Interfaces, Phys. Rev. Lett. 121, 204502 (2018).

[36] F. E. Culick, Comments on a ruptured soap film, J. Appl. Phys. 31, 1128 (1960).

[37] J. Eggers, J. R. Lister, and H. A. Stone, Coalescence of liquid drops, J. Fluid Mech. 401, 293 (1999).

[38] J. M. Montanero and A. M. Ganán-Calvo, Dripping, jetting and tip streaming, Rep. Prog. Phys. 83, 097001 (2020).

[39] R. J. Thomas, D. Webber, R. Hopkins, A. Frost, T. Laws, P. N. Jayasekera, and T. Atkins, The cell membrane as a major site of damage during aerosolization of escherichia coli, Appl. Environ. Microbiol. 77, 920 (2011).

[40] H. Zhen, T. Han, D. E. Fennell, and G. Mainelis, Release of free dna by membrane-impaired bacterial aerosols due to aerosolization and air sampling, Appl. Environ. Microbiol. 79, 7780 (2013).

[41] We approximate the characteristic stress $\sigma$ associated with a shell deformation $\delta$ through the force scaling: $F \sim \sigma D_{p}^{2} \sim E h^{2} \delta / D_{p}$. Here $D_{p}$ is the particle diameter, $E$ is the elastic modulus, and $h$ is the membrane thickness. Extending the scaling relation, the EDR in a liquid with viscosity $\mu$ is estimated as $\Phi \sim$ $\sigma^{2} / \mu \sim\left(E^{2} / \mu\right)\left(h / D_{p}\right)^{4}\left(\delta / D_{p}\right)^{2}$. A combination of AFM measurement and modeling have estimated that the cowpea chlorotic mottle virus (diameter $D_{p}=28, \mathrm{~nm}$, wall thickness $h=3.8 \mathrm{~nm}$ ) has an elastic modulus of $E \approx 150 \mathrm{MPa}$ and would be irreversibly damaged if deformed in excess of $\delta / D_{p} \sim 0.2$ [42]. These parameters suggest that the EDR for irreversible damage could be quite large; when considering water as the carrier fluid $\left(\mu=10^{-3} \mathrm{~Pa} \mathrm{~s}\right)$, the EDR from our scaling exceeds $3 \times 10^{14} \mathrm{~W} / \mathrm{m}^{3}$. However for a larger virus, such as influenza (elastic modulus of $E \approx 45 \mathrm{MPa}$, diameter $D_{p}=100 \mathrm{~nm}$, thickness $h=5 \mathrm{~nm}[43]$ ), our scaling suggests a threshold EDR closer to $10^{11} \mathrm{~W} / \mathrm{m}^{3}$.

[42] J. Michel, I. Ivanovska, M. Gibbons, W. Klug, C. Knobler, G. Wuite, and C. Schmidt, Nanoindentation studies of full and empty viral capsids and the effects of capsid protein mutations on elasticity and strength, Proc. Natl. Acad. Sci. U.S.A. 103, 6184 (2006).

[43] M. G. Mateu, Mechanical properties of viruses analyzed by atomic force microscopy: A virological perspective, Virus Res. 168, 1 (2012).

[44] C. W. Cooper, K. A. Aithinne, E. L. Floyd, B. S. Stevenson, and D. L. Johnson, A comparison of air sampling methods for clostridium difficile endospore aerosol, Aerobiologia 35, 411 (2019).

[45] D. A. Edwards, J. C. Man, P. Brand, J. P. Katstra, K. Sommerer, H. A. Stone, E. Nardell, and G. Scheuch, Inhaling to mitigate exhaled bioaerosols, Proc. Natl. Acad. Sci. U.S.A. 101, 17383 (2004). 